\title{
Limb salvage surgery with joint preservation for malignant humeral bone tumors: operative procedures and clinical application
}

Jie Zhao ${ }^{1,2}$, Ming $X u^{1}$, Kai Zheng ${ }^{1}$ and Xiuchun $\mathrm{Yu}^{1^{*}}$ (1)

\begin{abstract}
Background: However, the application of limb salvage with joint preservation is controversial. The purpose of this study is to propose a selection strategy of joint-sparing operative procedures for humeral malignancies based on tumor origin, site and bone strength.

Methods: The medical data of 28 patients with humeral malignancies treated at our institute from January 2010 to December 2016 were analyzed retrospectively. The patients had a median age of 51 years (range, 8-82 years). Bone strength scoring system was utilized to evaluated bone strength of the tumor. Four joint-sparing surgical methods were performed on selected patients. Evaluation of limb function was based on the Musculoskeletal Tumor Society scoring system. Two-sample t-test was used to compare patient group data such as bone strength score and postoperative Musculoskeletal Tumor Society score.

Results: The mean follow-up period for the 7 patients with primary malignancies was 45 months (range, 15-66 months). One patient died due to recurrence and lung metastasis, while the remaining 6 patients $(6 / 7,85.7 \%)$ survived without recurrence. For the 21 patients with metastases, 5 survived with tumors, with an average survival time of 25.8 months (range, 9-48 months). The rest died from progression of the primary tumors. The mean bone strength score for the biological reconstruction group and non-biological reconstruction group was respectively 9.7 \pm 1.3 and $12.9 \pm 1.2$. A significant difference between the 2 groups $(p<0.05)$ was found. Mean postoperative Musculoskeletal Tumor Society score was respectively $27.2 \pm 1.8$ and $26.1 \pm 1.7$ for the 2 groups. There was no significant difference between the 2 groups $(p>0.05)$. Non-oncological complications included fracture (1), aseptic loosening (1) and radial nerve injury (1).

Conclusions: Alcohol devitalized autograft replantation is applicable for diaphyseal humeral primary malignancies, with a good response to chemotherapy and a low bone strength score $(\leq 10)$. In situ microwave ablation is suitable for diaphyseal and (or) metaphyseal low-grade malignant bone tumors or metastases with a low bone strength score $(\leq 10)$. Intercalary prosthetic reconstruction is preferred for diaphyseal metastases with a high bone strength score $(>10)$.
\end{abstract}

Keywords: Joint preservation-limb salvage surgery-malignant humeral bone tumors-operative procedure

\footnotetext{
* Correspondence: 13969132190@163.com

1 Department of Orthopedics, The PLA 960th Hospital of China, 25\#, Shifan

Road, Jinan 250031, China

Full list of author information is available at the end of the article
}

(c) The Author(s). 2019 Open Access This article is distributed under the terms of the Creative Commons Attribution 4.0 International License (http://creativecommons.org/licenses/by/4.0/), which permits unrestricted use, distribution, and reproduction in any medium, provided you give appropriate credit to the original author(s) and the source, provide a link to the Creative Commons license, and indicate if changes were made. The Creative Commons Public Domain Dedication waiver (http://creativecommons.org/publicdomain/zero/1.0/) applies to the data made available in this article, unless otherwise stated. 


\section{Background}

The humerus is the third most common site for the development of primary malignant bone tumors, and is the second most common site affected by metastatic bone tumors involving long bones [1, 2]. As recent advancement of radiation therapy and chemotherapy, limb salvage procedures have been the main method of surgical treatment for humeral malignancies. Nowadays, more than $80 \%$ of the patients are treated with limb salvage surgery without critically compromising oncological principles [3]. Most humeral malignant bone tumors are located on the epiphysis and/or the metaphysis. Wide resection of the tumors often requires sacrifice of the native shoulder joint based on the principle of treatment for malignant bone tumors. However, the ideal surgical procedure for reconstruction after proximal humeral resection remains controversial. The common reconstruction methods include osteoarticular allografts, allograftprosthetic composites, endoprosthesis, clavicula pro humero, vascularized fibular grafts, extracorporeal inactivated autograft, or combined fibular graft with allograft/recycled autograft [4-10]. However, some humeral malignancies occur in the metaphysis or diaphysis, making it possible to preserve the adjacent joints.

However, there is no consistent application standards for various limb salvage operative procedures with joint preservation. We retrospectively reviewed medical records of 28 patients who had undergone joint-sparing limb salvage surgery for humeral malignant bone tumors at our institute. The purpose of this study is to propose a selection strategy of joint-preserving operative procedures for humeral malignant bone tumors based on tumor origin, site and bone strength.

\section{Methods}

Inclusion criteria were as follows: (i) diaphysis or metaphysis humeral malignant bone tumors not involving major blood vessels and nerves; (ii) primary malignant bone tumors sensitive to neoadjuvant chemotherapy; (iii) life expectancy of patients with humeral metastases $>3$ months; (iv) patients who underwent joint-sparing limb salvage surgery; (v) complete follow-up data. Exclusion criteria were as follows: (i) humeral malignant bone tumors involving the epiphysis; (ii) patients who underwent joint resection and reconstruction; (iii) incomplete follow-up data.

The clinical data of 28 eligible patients (12 males and 16 females) with humeral diaphysis or metaphysis malignant bone tumors treated at our institute between January 2010 and December 2016 were retrospectively analyzed (Table 1). The patients had a median age of 51 years (range, 8-82 years) at diagnosis. Pathological diagnosis of all patients was determined through aspiration biopsy. There were 7 patients with primary malignant bone tumors, 17 patients with metastatic bone tumors, and 4 patients with hematological malignancies. Apart from 3 chondrosarcoma patients, all patients with primary malignant bone tumors had received preoperative neoadjuvant chemotherapy. The chemotherapy regimens included cisplatin, doxorubicin and ifosfamide in accordance with a previously published report [11]. Joint-sparing limb salvage surgery was performed on patients who had an excellent response to chemotherapy. The degree of limb pain was judged using a visual analogue scale (VAS) and the patients were divided into mild, moderate and severe pain groups according to their VAS score.

The preoperative imaging data of all patients were comprehensively analyzed to assess tumor site, nature and bone strength. Tumor boundaries were identified using magnetic resonance imaging (MRI) data. The tumors were in the diaphysis and/or metaphysis, and did not invade beyond the epiphyseal line or plate. According to the Musculoskeletal Tumour Society (MSTS) resection classification system [12], the defects were classified as S4 (4 patients), S5 (20 patients), S45 (3 patients) and S45E1 (one patient). Based on Mirel's scoring system [13] and the invasion score for evaluating the extent of bone destruction through osteosarcoma [14], we made some improvements and proposed the bone strength scoring system. Five variables, including pain degree, tumor site, nature, length and transverse diameter, were utilized to evaluated the bone strength of the tumor segment (Table 2). The length and diameter of the tumors were classified as $<1 / 3, \geq 1 / 3$ and $\leq 2 / 3,>2 / 3$ of the length and diameter of the humeral diaphysis, respectively. Each variable was given 3 points, with a maximum score of 15 points. The higher the score, the lower the bone strength.

All patients underwent limb salvage surgery with joint preservation. In accordance with tumor origin, site and bone strength score, four different surgical methods with joint-sparing were performed in selected patients. As shown in Tables 1, 13 patients underwent biological reconstruction while 15 patients underwent non-biological reconstruction. Biological reconstruction methods included alcohol inactivated autograft replantation (AAR) with joint preservation (3) and in situ microwave ablation (MA) (10). Non-biological reconstruction methods included intercalary prosthetic reconstruction (9) and intramedullary nail or steel plate osteosynthesis with adjunctive bone cement (6).

Routine postoperative follow-up was performed on all patients. Oncological outcome, functional results and imaging findings were assessed and recorded at every follow-up visit. Evaluation of limb function was based on the Musculoskeletal Tumor Society (MSTS) scoring system for the upper extremity [15] (Table 1). 
Table 1 General information, bone strength score, surgical procedure, follow-up of 28 patients

\begin{tabular}{|c|c|c|c|c|c|c|c|c|}
\hline Case & Diagnosis & site & bone strength score & Surgical procedure & $\begin{array}{l}\text { Follow-up } \\
\text { (months) }\end{array}$ & MSTS score & Complication & Outcomes \\
\hline 1 & PNET & S5 & 10 & AAR & 28 & 24 & Recurrence, lung metastasis & Dead \\
\hline 2 & POS & S5 & 8 & AAR & 66 & 30 & & DFS \\
\hline 3 & ES & S5 & 9 & AAR & 63 & 30 & & DFS \\
\hline 4 & CS & S4 & 10 & MA & 54 & 29 & & DFS \\
\hline 5 & CS & S4 & 9 & MA & 30 & 27 & & DFS \\
\hline 6 & CS & S4 & 9 & MA & 60 & 27 & & DFS \\
\hline 7 & ES & S45E1 & 12 & MA & 15 & 29 & & DFS \\
\hline 8 & gastric cancer & S5 & 11 & MA & 15 & 27 & & Dead \\
\hline 9 & Bladder cancer & S5 & 8 & MA & 15 & 26 & & Dead \\
\hline 10 & Lung tumor cancer & S5 & 9 & MA & 6 & 27 & & Dead \\
\hline 11 & kidney cancer & S4 & 9 & MA & 18 & 26 & & Dead \\
\hline 12 & MM & S45 & 11 & MA & 12 & 26 & & SWT \\
\hline 13 & $\mathrm{NHL}$ & S45 & 11 & MA & 18 & 26 & & Dead \\
\hline 14 & Lung tumor cancer & S5 & 11 & PF & 18 & 26 & & Dead \\
\hline 15 & Breast cancer & S5 & 14 & $\mathrm{PF}$ & 21 & 27 & & Dead \\
\hline 16 & MM & S45 & 15 & PF & 9 & 25 & & SWT \\
\hline 17 & MM & S5 & 12 & PF & 42 & 24 & Fracture of internal fixation & Dead \\
\hline 18 & Esophageal cancer & S5 & 14 & INF & 9 & 27 & & Dead \\
\hline 19 & kidney cancer & S5 & 12 & INF & 24 & 25 & & Dead \\
\hline 20 & Breast cancer & S5 & 12 & $\mathbb{I P}$ & 18 & 22 & aseptic loosening & TBS \\
\hline 21 & Liver cancer & S5 & 14 & $\mathbb{I P}$ & 6 & 28 & & Dead \\
\hline 22 & Lung cancer & S5 & 14 & $\mathbb{I P}$ & 6 & 26 & Radial nerve injury & Dead \\
\hline 23 & Breast cancer & S5 & 14 & $\mathbb{I P}$ & 42 & 27 & & SWT \\
\hline 24 & Lung cancer & S5 & 13 & IP & 6 & 29 & & Dead \\
\hline 25 & Thyroid cancer & S5 & 13 & IP & 48 & 27 & & SWT \\
\hline 26 & Lung cancer & S5 & 12 & IP & 6 & 26 & & Dead \\
\hline 27 & kidney cancer & S5 & 12 & IP & 23 & 26 & & Dead \\
\hline 28 & Lung cancer & S5 & 11 & IP & 12 & 27 & & Dead \\
\hline
\end{tabular}

PNET primitive neuroectodermal tumor, POS parosteal osteosarcoma, ES Ewing sarcoma, CS chondrosarcoma, MM multiple myeloma, NHL non-Hodgkin lymphoma, $A A R$ alcohol inactivated autograft replantation, MA microwave ablation, PF plate fixation with bone cement, INF intramedullary nail fixation with bone cement, IP intercalary prosthesis, DFS disease-free survival, SWT survival with tumor

SPSS software (version 17.0, IBM, Armonk, NY, USA) was used to perform statistical analyses. We used chi-square test to compare count data such as gender. Measurement data are presented as mean \pm standard deviation. The measurement data were

Table 2 Bone strength scoring system

\begin{tabular}{llll}
\hline Variable & \multicolumn{3}{c}{ Score } \\
& 1 & 2 & 3 \\
\hline Pain & Mild & Moderate & Severe \\
site & Distal humerus & Diaphysis & Proximal humerus \\
Nature & Osteogenic & Mixed & Osteolytic \\
Length & $<1 / 3$ & $\geq 1 / 3, \leq 2 / 3$ & $>2 / 3$ \\
Transverse diameter & $<1 / 3$ & $\geq 1 / 3, \leq 2 / 3$ & $>2 / 3$ \\
\hline
\end{tabular}

treated with normality test. Mann-Whitney $\mathrm{U}$ test was used to compare age difference which not conformed to normal distribution. Bone strengh score and postoperative MSTS score were accorded with the normal distribution. Two-sample T-test was used to compare them between the biological reconstruction group and non-biological reconstruction group. A two-tailed $P$ value of $<0.05$ was considered as statistically significant.

The research has been performed in accordance with the declaration of Helsinki. Our study was approved by the Ethics Committee of the PLA 960th hospital. All adult patients and parents for children who participate in the study provided written informed consent. 


\section{Results}

Postoperative follow-up was conducted on all patients. The mean follow-up duration for the 7 patients with primary malignant bone tumors was 45 months (range, 1566 months). One patient $(1 / 7,14.3 \%)$ who had undergone alcohol inactivated autograft replantation had local recurrence 7 months after the initial operation, and eventually died of lung metastasis. The remaining 6 patients who had a primary malignant bone tumor were alive and tumor-free. 5 of the 21 patients with metastatic or hematological malignancies were alive with the tumor, with an average survival period of 25.8 months (range, 948 months). The remaining 16 patients died of tumor progression by the end of the follow-up period.

There was no significant difference in gender and age between the two groups $(p>0.05)$.The average bone strength score of patients included in the current study was $11.4 \pm 2.0$ (range, 8-15). The mean bone strength score of the biological reconstruction group and nonbiological reconstruction group was $9.7 \pm 1.3$ and $12.9 \pm$ 1.2 , respectively. There was significant statistical difference for bone strength score between the two groups ( $p$ $<0.05$, Table 3).

The mean postoperative MSTS score of the 28 patients was $26.6 \pm 1.8$ (range, 22-30). Postoperative limb function was able to meet the daily needs of the patients. The average MSTS score of the biological reconstruction group and non-biological reconstruction group was 27.2 \pm 1.8 and $26.1 \pm 1.7$, respectively. There was no significant statistical difference for mean postoperative MSTS score between the two groups ( $p>0.05$, Table 3$)$.

Non-oncological complications were encountered in 3 of the 28 patients $(3 / 28,10.7 \%)$, and included fracture and plate breakage in one patient (3.6\%), aseptic loosening in another (3.6\%), and radial nerve injury in yet another (3.6\%). The patient with fracture and plate breakage was treated by removal of internal fixation and reconstruction of bone defect with intercalary prosthesis (Fig. 1). The patient with radial nerve injury was able to return to normal function after nerve electric stimulation treatment. The patient with aseptic loosening of segmental prosthesis received conservative treatment (Fig. 2).

\section{Discussion}

The humerus is one of the most common sites of primary bone sarcomas and metastasis. At present, limb salvage has become the main surgical approach for the treatment of humeral malignant bone tumors, but the optimal procedure for preserving the adjacent shoulder and elbow joints remain a subject of debate. San-Julian et al. were the first to divide juxtarticular osteosarcoma in children into three types based on the relationship between the tumor border and the epiphyseal plate [16]. We once reported of the clinical application of alcoholinactivated autograft replantation with articulation preservation for San-Julian type I osteosarcoma of the distal femur [11]. However, joint preservation for San-Julian type II tumors is still controversial. Some researchers believe that a safe margin cannot be obtained in such a situation [17]. By analyzing the impacts of tumor location, nature and bone destruction on a selection of limb salvage procedures, we previously found that biological reconstruction methods with joint preservation are feasible for diaphysis or metaphysis sarcomas with a low invasion score $(\leq 8)$ [14]. Based on a comprehensive evaluation of tumor origin, site and bone strength in this study, we put forward a selection strategy of jointpreserving limb salvage surgery for malignant humeral bone tumors.

Extracorporeal devitalized autograft replantation is an effective biological reconstruction method used to repair segmental bone defects following tumor resection of long bones, especially in countries where it is difficult to obtain massive allografts. The advantages of alcohol devitalization are low-cost, easy operation, and excellent shape matching ability [11]. However, there is a trade-off between the thoroughness of tumor devitalization and the loss of bone strength when a tumor segment is inactivated in vitro. We previously reported on the application of alcohol-inactivated autograft replantation for the treatment of a diaphysis humeral Ewing sarcoma patient [18]. Complete bone union was seen at the host-graft interface 5 months after operation, and shoulder and elbow joint function have returned to normal. There were no complications such as fracture of inactivated bone, nonunion, recurrence or lung metastasis that

Table 3 Comparisons between biological reconstruction and non-biological reconstruction

\begin{tabular}{llll}
\hline Comparative features & biological reconstruction & non-biological reconstruction & P value \\
\hline Gender(F/M) & $7 / 6$ & $9 / 6$ & 1.000 \\
Age(y) & $56 \pm 16.8$ & $59 \pm 10.6$ & 0.289 \\
Bone strength score & $9.7 \pm 1.3$ & $12.9 \pm 1.2$ & 0.000 \\
MSTS score & $27.2 \pm 1.8$ & $26.1 \pm 1.7$ & 1.097 \\
\hline
\end{tabular}

MSTS Musculoskeletal Tumor Society

$p<0.05$ indicated a significant difference between the two groups 

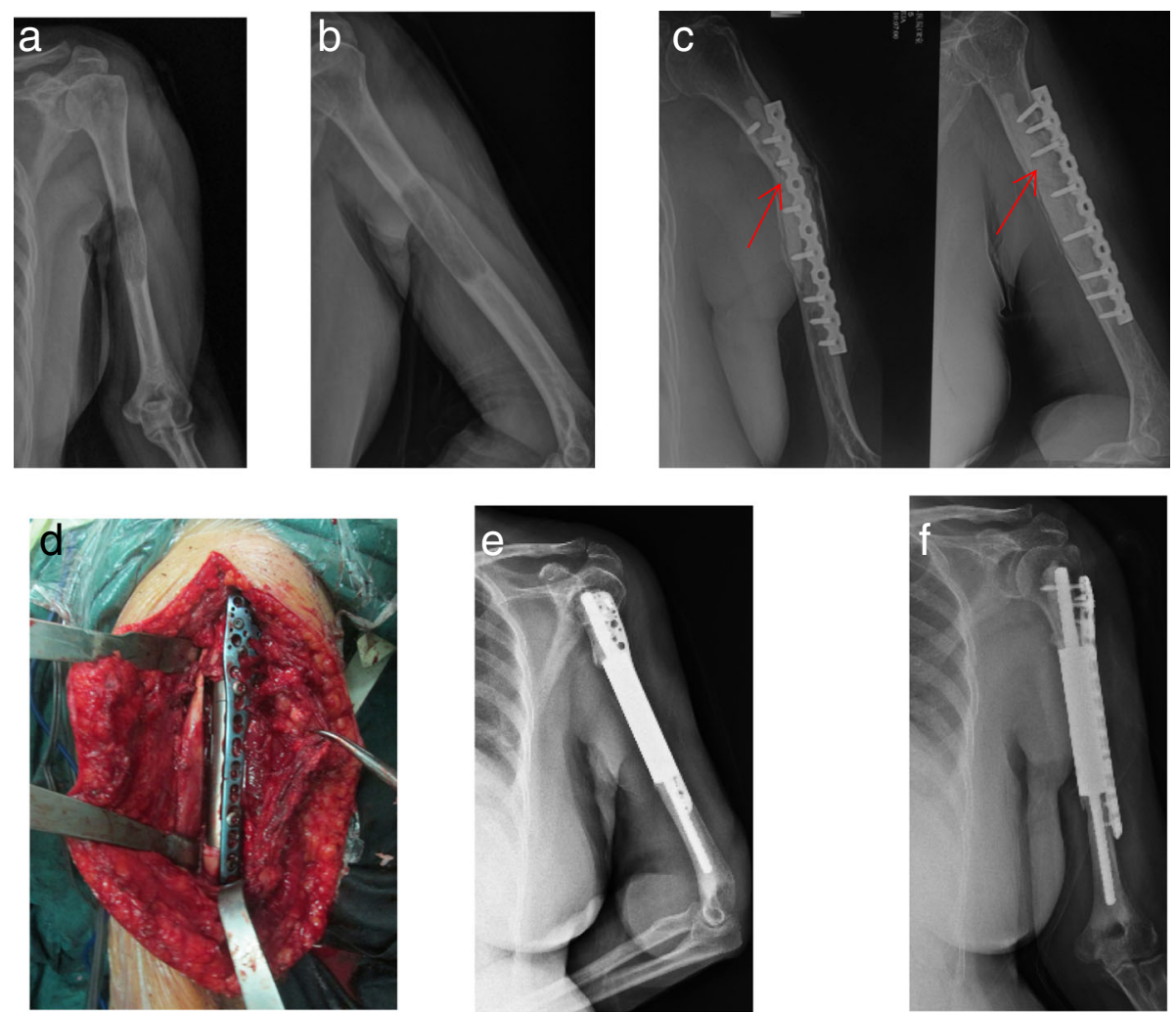

Fig. 1 Case 20, a 62-y old female patient with osteolytic lesion due to multiple myeloma in the left humerus. The preoperative bone strength score is 12. a, b The tumor was located in the left humerus shaft (S5). $\mathbf{c}$ The patient underwent resection of the tumor and steel plate osteosynthesis with adjunctive bone cement. Fracture and screw breakage occurred 33 months after surgery (arrow). $\mathbf{d}$ Then, this patient underwent removement of the internal fixation and intercalary prosthetic reconstruction with an additional plate. e, $\mathbf{f}$ Postoperative $\mathbf{X}$-ray indicated that intercalary prosthesis was in excellent position

occurred during the follow-up period of more than 5 years (Fig. 3). In our study, 3 patients with primary bone sarcomas of the humeral shaft underwent alcoholinactivated autograft replantation. They received two courses of neoadjuvant chemotherapy prior to operation. Their response to chemotherapy was relatively satisfactory and preoperative MRI data demonstrated that the tumor did not invade the peripheral blood vessels and nerves. Based on the bone strength scoring system, the average bone strength score of the three cases was 9 (range, 8-10), indicating that the extent of bone destruction was low. Consequently, alcohol inactivated autograft replantation along with joint preservation was performed on the 3 patients. The mean postoperative MSTS score was $28(28 / 30,93.3 \%)$ at the end of the follow-up period. Function of the affected limbs had evidently improved by preserving the native shoulder and elbow joint. A retrospective analysis of 191 patients with bone tumors who were treated with the alcohol inactivated autograft replantation showed that the overall local recurrence rate was $26.7 \%$, lung metastasis rate was $27.2 \%$, and bone nonunion rate was $17.3 \%$ [19]. One patient suffered local recurrence, which may have been related to inadequate resection margins. The patient died of lung metastasis 28 months after surgery. The average bone healing duration was 6.8 months (range, 6-10 months), which is similar to that of massive allografts [10]. A previous study had showed that the fracture rate of alcoholinactivated autografts can be as high as $20.4 \%$ [19]. In order to reduce the incidence of fracture, we carefully evaluated the mechanical strength of the tumor segments using the bone strength scoring system prior to operation. Reconstruction of bone defects following tumor resection using alcohol-devitalized autografts is not recommended for severe osteolytic lesions with a bone strength score of $>10$. No devitalized bone fractures were observed in selected patients.

In situ microwave ablation was performed on 10 patients with malignant bone tumors in the diaphysis and/ or metaphysis part of the humerus. Among them, there were 3 cases of chondrosarcoma, 6 cases of metastatic bone tumors, and 1 case of Ewing sarcoma. Taking the Ewing sarcoma patient as an example, the tumor border was not invaded beyond the epiphyseal plate, and obvious tumor calcification was observed after chemotherapy. This patient underwent in situ microwave ablation 

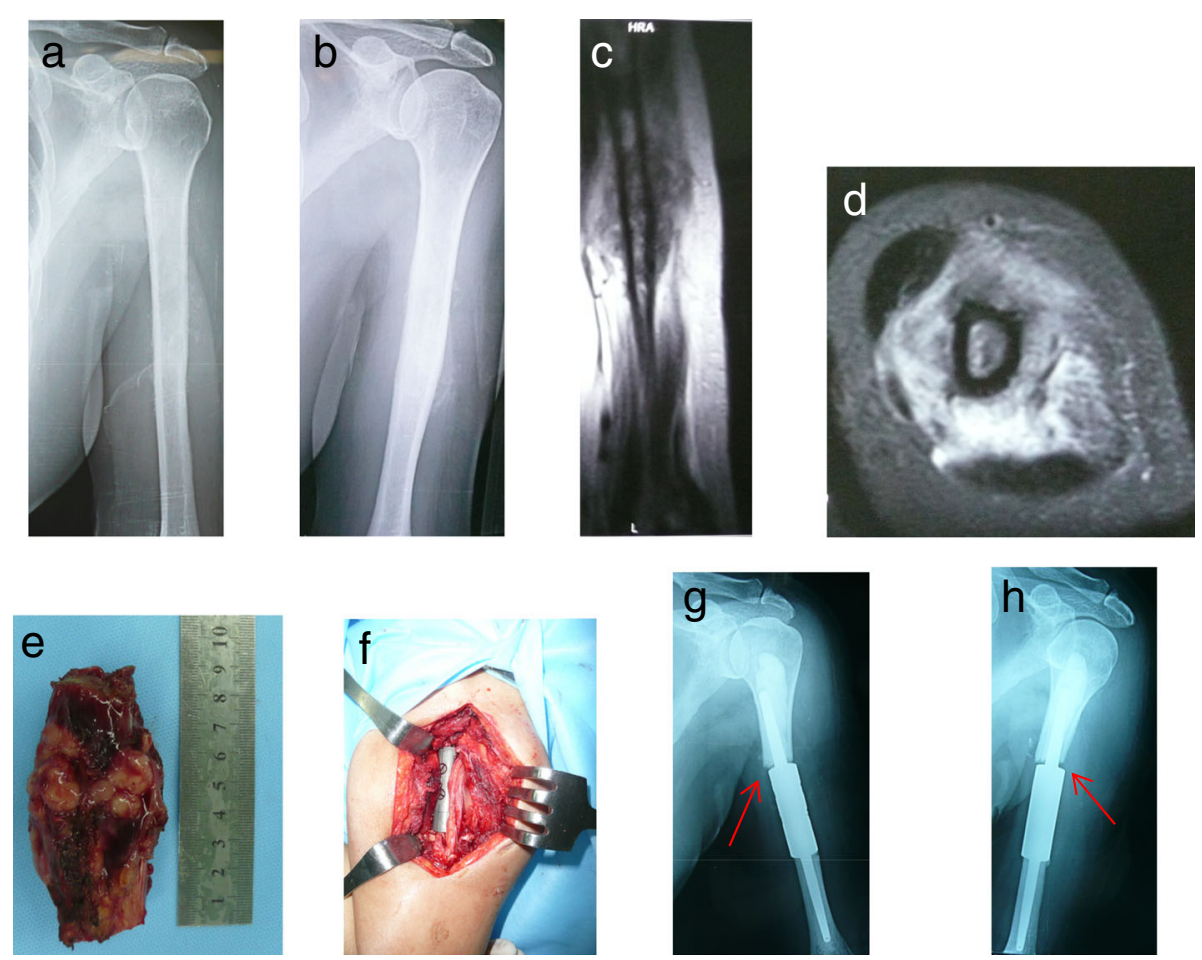

Fig. 2 Case 23, a 65-y old female patient with metastatic tumor secondary to breast cancer. The bone strength score is 12 . $\mathbf{a}, \mathbf{b}$ The preoperative $\mathrm{X}$-ray showed that the osteolytic destruction was located in the diaphysis of left humerus( 55 region). $\mathbf{c}$, $\mathbf{d}$ The preoperative MRI indicated that the intramedullary destructive signals and soft-tissue swelling. $\mathbf{e}, \mathbf{f}$ This patient underwent resection of the tumor and intercalary prosthetic reconstruction. $\mathbf{g}, \mathbf{h}$ The proximal stem of the prosthesis occurred aseptic loosening 3 months after operation(arrow)

and internal fixation. No complications of local recurrence and fracture occurred up to 15 months after surgery (Fig. 4). Fan et al. [20] reported a local recurrence rate of $9.8 \%$ in patients with malignant extremity bone tumors treated with microwave inactivation, and the rate of mechanical complications was $2.6 \%$. At the last follow-up visit, the mean MSTS score of the 10 patients was 27 (range, 26-29), which is comparable to values published in existing literature [20]. Fan et al. [20] reported that the over 3-year survival rate of high-grade and low-grade malignancies are 59.1 and $88.7 \%$, respectively. This significant difference indicates that microwave ablation might be more suitable for low-grade malignant bone tumors. $\mathrm{Hu}$ et al. [21] found that high cortical brittleness and low biomechanical properties induced by microwave hyperthermia could increase the risk of postoperative pathological fractures. The mean bone strength score of patients who underwent in situ microwave ablation was 9.9 (range, 8-12), indicating that the extent of bone destruction is not severe. Thus, they underwent in situ microwave inactivation and internal fixation. After curettage of inactivated tumor tissue, bone cement was used to fill the tumor cavity and a steel plate was used to strengthen fixation. After operation, the affected extremity was immobilized for 6 weeks. The 10 patients were followed up for 6-60 months, and no postoperative fracture was found. In brief, in situ microwave ablation is suitable for patients with humeral malignancies (low-grade malignant bone tumors or metastatic tumors) in the diaphysis and/or metaphysis with a low bone strength score $(\leq 10)$.

There are many methods of reconstruction of segmental bone defects from intercalary resection of metastatic tumors, including intercalary prosthesis, massive allografts, intramedullary nail and plate combined with bone cement [22-25]. However, devitalized autografts are not suitable for the reconstruction of metastatic diaphyseal defects with a high bone strength score $(>10)$. During the early stage of this study, intramedullary nail or plate combined with bone cement was usually used for the reconstruction of segmental defects following the resection of metastatic tumors. The mean postoperative MSTS score of these patients was 25.7 (range, 24-27), indicating $85.7 \%$ normal function. One of the six cases suffered from fracture and screw breakage 33 months after operation. Then the patient underwent removal of the plate combined with bone cement and reconstruction using a custom-made intercalary prosthesis (Wego, Beijing, China) (Fig. 1). Some biomechanical studies have demonstrated that intercalary prosthesis performs better in various types of loading (four-point bending, torsion, compression) when compared with other fixation 

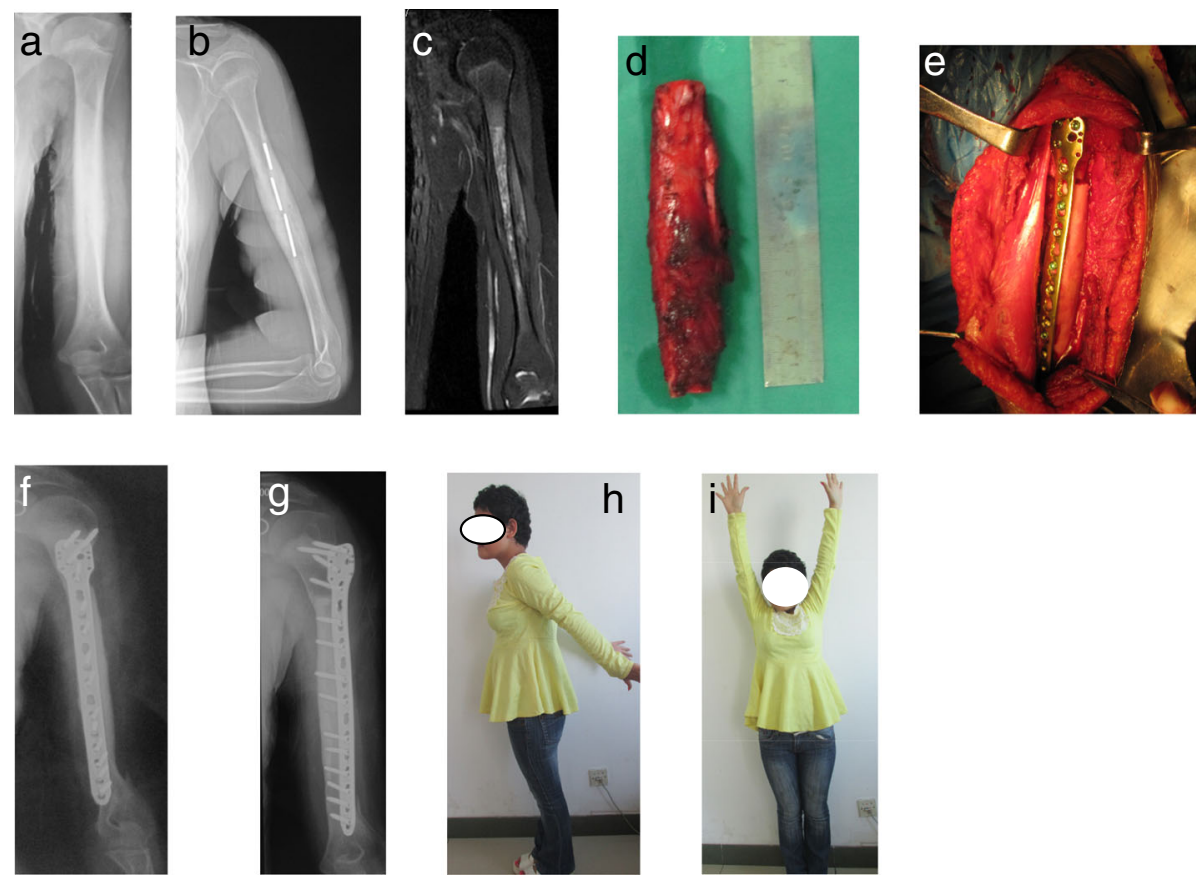

Fig. 3 Case 3, a 11-y old female patient with Ewing sarcoma in the diaphysis of left humerus (S5). The preoperative bone strength score is 9 . $\mathbf{a}$, b The preoperative X-ray showed that osteogenic destruction (a) before chemotherapy and obvious calcification (b) after chemotherapy. c The preoperative MRI showed that abnormal intramedullary high signal with clear borders on T2WI. $\mathbf{d}$, e This patient underwent resection of the tumor segment and replantation of the alcohol-inactivated autograft followed by plate internal fixation. $\mathbf{f}, \mathbf{g} 5$ years after operation,the X-ray indicated that internal fixation was in excellent position with no local tumor recurrence. $\mathbf{h}$, i Postoperative shoulder joint function returned to normal range

methods [22, 24]. Intercalary prosthetic reconstruction is of benefit to patients with metastatic diaphyseal tumors due to the advantages of immediate stability, preservation of adjacent joints and an early return of function $[23,26]$. After 2013, intercalary prosthesis was used to reconstruct segment skeletal defects instead of rush rod or plate augmented with bone cement. The mean postoperative MSTS score of patients who underwent intercalary prosthetic reconstruction was 26.4 (range, 22-29), indicating $88 \%$ normal function. However, early loosening has been reported to occur in $9.5-30.8 \%$ of cases $[27,28]$. Some authors believe that high rotational stress in the upper extremity may lead to early loosening [27, 29]. Among our series of patients, one case of aseptic loosening of the implant with short intramedullary fixation was found 3 months after operation. This may have been related to high rotational stress and traction in the upper limb. Some researchers believe that the length of the remaining bone in prosthesis fixation should be $>5 \mathrm{~cm}[29,30]$. Some authors have suggested that additional extracortical plates should be added to short-segment intramedullary fixation $<5 \mathrm{~cm}$ or bone defects $>10 \mathrm{~cm}[31,32]$. A biomechanical analysis of a novel intercalary prosthesis for humeral diaphyseal segmental defect reconstruction showed that intercalary prosthesis with plate fixation improves the rigidity of antitension and anti-torsion, and diminishes the risk of early loosening and dislocation [33]. In order to reduce the incidence of prosthetic loosening, we used a custom-made intercalary prosthesis and added a PHILOS plate, a straight plate, or a condylar plate for the reconstruction of segmental defects in the proximal, middle, or distal diaphysis humerus, respectively [34].

Wafa et al. [35] reported a mean MSTS score of 24.8 \pm 2.6 (range, 18-28) for 28 patients who survived their disease for more than 12 months after total humeral endoprosthetic replacement. In the present study, the mean MSTS score for the 28 patients who underwent joint-sparing limb salvage was $26.6 \pm 1.8$ (range, 22-30), indicating excellent limb function. All patients had returned to their preoperative daily activities at the last follow-up visit.

There are some limitations and shortcomings in this study. First, the number of cases of different operative procedures is very small due to the infrequent application of joint preservation. Second, this is a single center retrospective study. Up to a certain extent, selection of operative procedures was affected by personal experience and preference. There were also no direct comparisons with other reconstructive methods such as massive allografts or fibular grafts. Third, only a few cases was followed up for more than 5 years. 

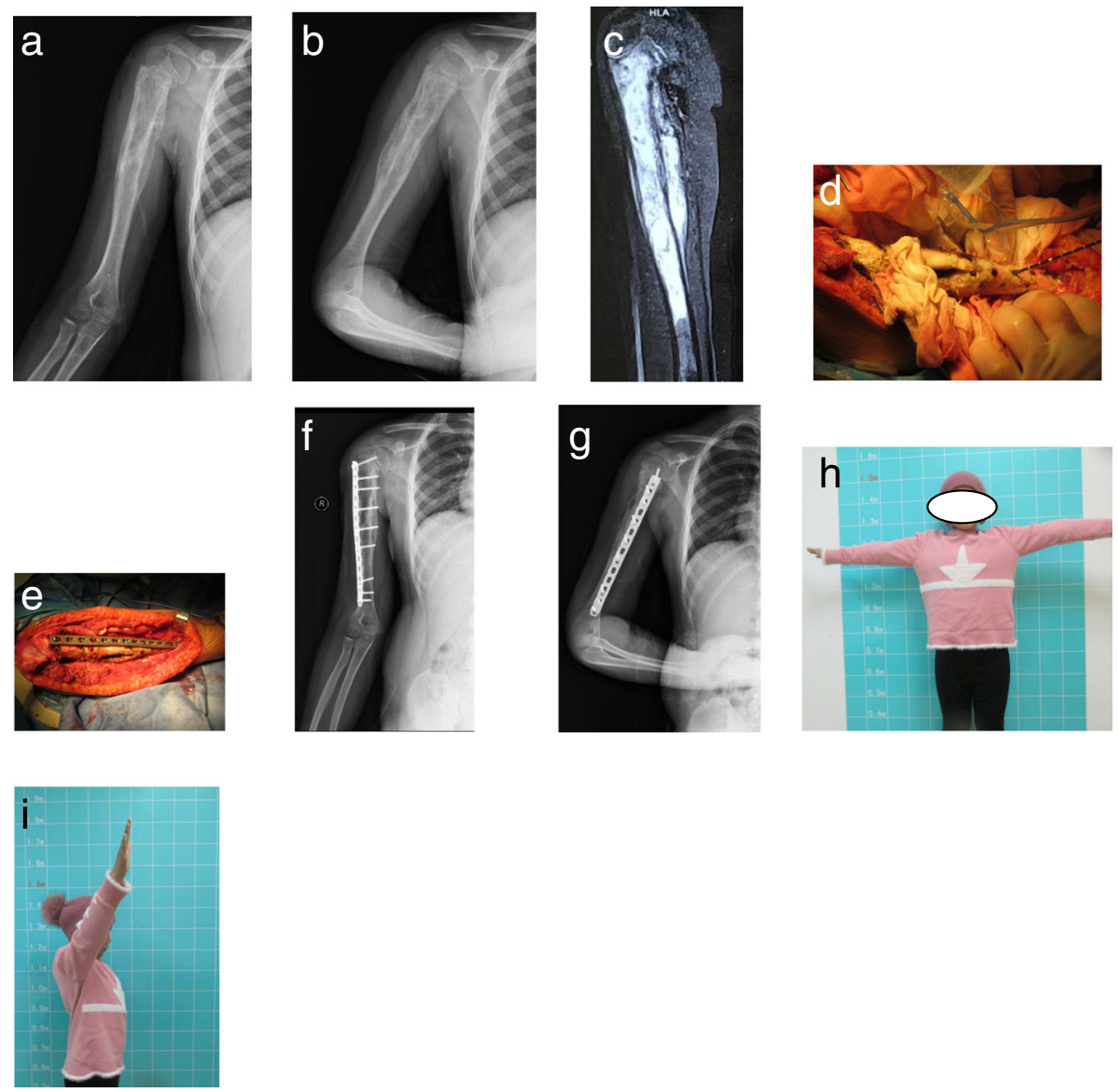

Fig. 4 Case 7, a 8-y old female patient with Ewing sarcoma in the right humerus(S45E1). The preoperative bone strength score is 12. a, b The post-chemotherapy X-ray showed that obvious calcification after chemotherapy. c The post-chemotherapy MRI showed that tumor did not invade epiphyseal plate with a clear bounder. $\mathbf{d}$, e This patient underwent in situ microwave ablation and plate internal fixation. $\mathbf{f}, \mathbf{g} 15$ months after operation,the X-ray indicated that internal fixation was in good position and no local recurrence of tumor occurred. $\mathbf{h}, \mathbf{i}$ The patient received excellent abduction and lifting function of shoulder joint at the last follow-up

\section{Conclusions}

Selection of the joint-preserving limb salvage operative procedure should be based on tumor origin, site and bone strength. Alcohol devitalized autograft replantation is applicable for diaphyseal humeral primary malignancies with a good response to chemotherapy and a low bone strength scores $(\leq 10)$. In situ microwave ablation is suitable for diaphyseal and (or) metaphyseal low-grade malignant bone tumors or metastases with a low bone strength scores $(\leq 10)$. Intercalary prosthetic reconstruction is preferred for diaphyseal metastases with a high bone strength scores $(>10)$.

\section{Abbreviations}

AAR: Alcohol inactivated autograft replantation; MA: Microwave ablation; MRI: Magnetic resonance imaging; MSTS: Musculoskeletal Tumor Society; VAS: Visual analogue scale

\section{Acknowledgments}

Not applicable

\section{Authors' contributions}

YXC conceived and designed the study. ZJ, XM, and ZK performed the study. ZJ drafted the manuscript. ZJ, YXC reviewed and edited the manuscript. All authors provided intellectual input to the study and approved the final version of the manuscript.

\section{Funding}

No fundings

\section{Availability of data and materials}

The datasets generated during and/or analysed during the current study are not publicly available due they are paper document in chinese language store in our hospital archives but are available from the corresponding author on reasonable request.

\section{Ethics approval and consent to participate}

Our study was approved by the Ethics Committee of the PLA 960th hospital. All adult patients and parents for children who participate in the study provided written informed consent. A copy of the consent form is available for review.

\section{Consent for publication}

Written informed consent for publication of the pictures was obtained from parents of the children who appear in the figures. And the participants (included adult patients and parents for the children patients) gave written 
informed consent for the publication of their details in the manuscript. A copy of the written informed consent is available for review by the editor.

\section{Competing interests}

No benefits in any form have been received or will be received from a commercial party related directly or indirectly to the subject of this research.

\section{Author details}

'Department of Orthopedics, The PLA 960th Hospital of China, 25\#, Shifan Road, Jinan 250031, China. ${ }^{2}$ First Clinical Medical College, Shandong University of Traditional Chinese Medicine, 4655\#, Daxue Road, Jinan 250355, China.

Received: 12 February 2019 Accepted: 21 May 2019 Published online: 30 May 2019

\section{References}

1. Damron TA, Ward WG, Stewart A. Osteosarcoma, chondrosarcoma, Ewing's sarcoma: National Cancer Date Base Report. Clin Orthop Relat Res. 2007; 459(3):40-7. https://doi.org/10.1097/BLO.0b013e318059b8c9.

2. Frassica FJ, Frassica DA. Evaluation and treatment of metastases of the humerus. Clin Orthop Relat Res. 2003;(415 Suppl):S212-8. https://doi.org/10. 1097/01.blo.0000093052.96273.a7

3. Weber KL. What's new in musculoskeletal oncology. J Bone J Surg Am. 2005;87(6):1400-10. https://doi.org/10.2106/JBJS.I.00375.

4. Teunis T, Nota SP, Hornicek FJ, Schwab JH, Lozano-Calderón SA. Outcome after reconstruction of the proximal humerus for tumor resection: a systematic review. Clin Orthop Relat Res. 2014;(7):2245-53. https://doi.org/ 10.1007/s11999-014-3474-4.

5. Bus MP, van de Sande MA, Taminiau AH, Dijkstra PD. Is there still a role for osteoarticular allograft reconstruction in musculoskeletal tumour surgery? A long-term follow-up study of 38 patients and systematic review of the literature. Bone Joint J. 2017;(4):522-30. https://doi.org/10.1302/0301-620X. 99B4.BJJ-2016-0443.R2.

6. Natarajan M, Sameer M, Kunal D, Balasubramanian N. Custom-made endoprost-.hetic total humerus reconstruction for musculoskeletal tumours. Int Orthop. 2012;36(1):125-9. https://doi.org/10.1007/s00264-011-1316-5.

7. Barbier D, De Billy B, Gicquel P, Bourelle S, Journeau P. Is the clavicula pro humero technique of value for reconstruction after resection of the proximal humerus in children? Clin Orthop Relat Res. 2017;475(10):2550-61. https://doi.org/10.1007/s11999-017-5438-y.

8. Muramatsu K, Fukano R, Ihara K, Iwanaga R, Taguchi T. Reconstruction of the proximal humerus by combined use of extracorporeally-irradiated osteochondral graft and free vascularized fibula following resection of Ewing sarcoma. J Plast Reconstr Aesthet Surg. 2010;63(12):2177-80. https:// doi.org/10.1016/j.bjps.2010.03.008.

9. Ihara K, Doi K, Yamamoto M, Kawai S. Free vascularized fibular graft for large bone defect in the extremities after tumor excision. J Reconstr Microsurg. 1998;14:371-6. https://doi.org/10.1055/s-2007-1000193.

10. Li J, Wang Z, Pei G, Guo Z. Biological reconstruction using massive bone allograft with intramedullary vascularized fibular flap after intercalary resection of humeral malignancy. J Surg Oncol. 2011;104(3):244-9. https:// doi.org/10.1002/jso.21922.

11. Yu X, Xu S, Xu M, Liu X, Song R, Fu Z. Alcohol-inactivated autograft replantation with joint preservation in the management of osteosarcoma of the distal femur: a preliminary study. Oncol Res Treat. 2014;37:554-60. https://doi.org/10.1159/000367799.

12. Enneking W, Dunham W, Gebhardt M, Malawar M, Pritchard D. A system for the classification of skeletal resections. Chir Organi Mov. 1990:75:217-40.

13. Mirels H. Metastatic disease in long bones: a proposed scoring system for diagnosing impending pathologic fractures. Clin Orthop Relat Res. 1989; (249):256-64.

14. Chen $Y, Y u X, X u S, X u M$, Song R. Impacts of tumor location, nature and bone destruction of extremity osteosarcoma on selection of limb salvage operative procedure. Orthop Surg. 2016:8:139-49. https://doi.org/10.1111/os.12237.

15. Enneking WF, Dunham W, Gebhardt MC, Malawar M, Pritchard DJ. A system for the functional evaluation of reconstructive procedures after surgical treatment of tumors of the musculoskeletal system. Clin Orthop Relat Res. 1993;(286):241-6. https://doi.org/10.1097/00003086-199301000-00035.

16. San-Julian M, Aquerreta JD, Benito A, Cañadell J. Indications for epiphyseal preservation in metaphyseal malignant bone tumors of children: relationship between image methods and histological findings. J Pediatr Orthop. 1999;19(4):5438. https://doi.org/10.1097/00004694-199907000-00025.

17. Manfrini M, Gasbarrini A, Malaguti C, Ceruso M, Innocenti M, Bini S, Capanna R, Campanacci M. Intraepiphyseal resection of the proximal tibia and its impact on lower limb growth. Clin Orthop Relat Res. 1999;358:111-9.

18. Wang B, Yu X, Xu S, Xu M, Zheng K, Liu J. Alcohol-inactivated autograft replantation with joint preservation in the management of Ewing sarcoma of the humeral shaft: a case report and literature review. Chin J Shoulder Elb (Electronic Edition). 2015;3(2):46-50. https://doi.org/10.3877/cma.j.issn. 2095-5790.2015.02.009

19. Ding Y, Niu X, Liu W, Zhang Q, Hao L, Liu W, Yu F, Yang F. Excision-alcoholizationreplantation method in management of bone tumors. Chin J Orthop. 2011;31(6): 652-7. https://doi.org/10.3760/cma.j.issn.0253-2011.06.017.

20. Fan QY, Zhou Y, Zhang M, Ma B, Yang T, Long H, Yu Z, Li Z. Microwave ablation of malignant extremity bone tumors. SpringerPlus. 2016:5(1):13739. https://doi.org/10.1186/s40064-016-3005-8.

21. Hu Y, Wang J, Lu S, Zhao L, Bao S. The effects of microwave heating on the biomechanics of osseous tissue. Chin J Orthop. 1997;17(10):645-8.

22. Chin HC, Frassica FJ, Hein TJ, Shives TC, Pritchard DJ, Sim FH, Chao EY. Metastatic diaphyseal fractures of the shaft of the humerus. The structural strength evaluation of a new method of treatment with a segmental defect prosthesis. Clin Orthop Relat Res. 1989:(248):231-9.

23. Benevenia J, Kirchner R, Patterson F, Beebe K, Wirtz DC, Rivero S, Palma M, Friedrich M. Outcomes of a modular intercalary endoprosthesis as treatment for segmental defects of the femur, tibia, and humerus. Clin Orthop Relat Res. 2016;474(2):539-48. https:/doi.org/10.1007/s11999-015-4588-z.

24. Dijkstra S, Stapert J, Boxma H, Wiggers T. Treatment of pathological fractures of the humeral shaft due to bone metastases: a comparison of intramedullary locking nail and plate osteosynthesis with adjunctive bone cement. Eur J Surg Oncol. 1996;22(6):621-6. https://doi.org/10.1016/s07487983(96)92450-6.

25. Makley JT. The use of allografts to reconstruct intercalary defects of long bones. Clin Orthop Relat Res. 1985;(197):58-75.

26. Zhang J, Wang F, Hu Y. Reconstruction of humeral shaft defect with an intercalary endoprosthesis following resection of tumor. Orthop Surg. 2018; 10(3):281-4. https://doi.org/10.1111/os.12391.

27. McGrath A, Sewell MD, Hanna SA, Pollock RC, Skinner JA, Cannon SR, Briggs TW. Custom endoprosthetic reconstruction for malignant bone disease in the humeral diaphysis. Acta Orthop Belg. 2011;77(2):171-9.

28. Damron TA, Leerapun T, Hugate RR, Shives TC, Sim FH. Does the second generation intercalary humeral spacer improve on the first? Clin Orthop Relat Res. 2008;466(6):1309-17. https://doi.org/10.1007/s11999-008-0246-z.

29. AhImann ER, Menendez LR. Intercalary endoprosthetic reconstruction for diaphyseal bone tumours. J Bone Joint Surg Br. 2006;88(11):1487-91. https:// doi.org/10.1302/0301-620X.88B11.18038.

30. Abudu A, Carter SR, Grimer RJ. The outcome and functional results of diaphyseal endoprostheses after tumour excision. J Bone Joint Surg Br. 1996;78(4):652-7.

31. Sewell MD, Hanna SA, McGrath A, Aston WJ, Blunn GW, Pollock RC, Skinner JA, Cannon SR, Briggs TW. Intercalary diaphyseal endoprosthetic reconstruction for malignant tibial bone tumors. J Bone Joint Surg Br. 2011; 93:1111-7. https://doi.org/10.1302/0301-620X.93B8.25750.

32. Huang H, Hu Y, Lun D, Miao J, Wang F, Yang X, Ma X. Outcomes of intercalary prosthetic reconstruction for pathological Diaphyseal femoral fractures secondary to metastatic tumors. Orthop Surg. 2017;9(2):221-8. https://doi.org/10.1111/os.12327.

33. Zhao L, Tian D, Wei Y, Zhang J, Di Z, He Z, Hu Y. Biomechanical analysis of a novel intercalary prosthesis for humeral diaphyseal segmental defect reconstruction. Orthop Surg. 2018;10(1):23-31. https://doi.org/10.1111/os.12368.

34. Zhao J, Xu M, Zheng K, Hu Y, Wang F, Yu X. Intercalary prosthetic reconstruction for pathologic diaphyseal humeral fractures due to metastatic tumors:outcomes and improvements. J Shoulder Elb Surg. 2018; 27(11):2013-20. https://doi.org/10.1016/j.jse.2018.03.027.

35. Wafa H, Reddy K, Grimer R, Abudu A, Jeys L, Carter S, Tillman R. Does Total humeral Endoprosthetic replacement provide reliable reconstruction with preservation of a useful extremity? Clin Orthop Relat Res. 2015;473(3):91725. https://doi.org/10.1007/s11999-014-36.

\section{Publisher's Note}

Springer Nature remains neutral with regard to jurisdictional claims in published maps and institutional affiliations. 